DISKUSSIONSFORUM

\section{Mit grüner Biotechnologie zum größten Glück der größten Zahl?}

Ein Gedankenspiel zur doppelten Profitabilität gentechnisch veränderter Pflanzen

\author{
von Christian Berkenkopf, Ruhr-Universität \\ Bochum
}

Hinsichtlich der Frage einer Technikfolgenabschätzung ist zu diskutieren, ob der Anbau von gentechnisch veränderten Pflanzen überhaupt zulässig ist. Viel ist in diesem Feld bereits geschrieben worden, doch wird zumeist ausgeblendet, dass gentechnisch veränderte Pflanzen in globaler Perspektive bereits in großem Stil angebaut werden. Dieser Essay stellt die provokante Frage, was gedacht werden muss, damit der Anbau gentechnisch veränderter Pflanzen doppelt profitabel gestaltet werden kann: einerseits mit ökonomischem, andererseits mit wohlstandsorientiertem Nutzen. Die Überlegungen zu einem globalen „Health Impact Fund“ sollen als Modell für die grüne Biotechnologie adaptiert und diskutiert werden.

\section{Grüne Biotechnologie als Thema der Ethik}

Die ethischen Fragen zur grünen Biotechnologie sind komplex, und es ist nicht einfach, sich in der Debatte um eine Technik zu verorten, von der sich die Befürworter „das Paradies“ versprechen, ,während die Gegner die Hölle prophezeien“ (Korthals 2003, S. 354). Denn Gentechnik in der Pflanzenzüchtung stellt für den einen eine Verbesserung der Natur dar, für den anderen ist sie eine unzulässige Grenzüberschreitung. Eindrucksvoll liest sich in diesem Zusammenhang die Übersicht zu den unterschiedlichen Begründungsansätzen ethischer Argumentation in Christian Kummers Ausführungen zur Pflanzenwürde. Demnach sind holistische, anthropo-, ratio-, patho-, bio- und theozentrische Begründungen sowie Ansätze in Orientierung an der Kategorie pflanzlicher ,Natürlichkeit' letztlich nichts anderes als egozentrisch begründete Denkfiguren: „Ich selber werde im Umgang mit meiner Zimmerpflanze zum moralischen Objekt, weil es gut für mich ist, im Eingehen auf ihre Bedürfnisse Tugenden wie Sorgfalt und Rücksichtnahme zu (re-)aktivieren“" (Kummer 2013, S. 29).

Fragen wir einmal anders und nehmen die Beobachtung Kummers auf. Weil offenbar die Diskussion ohne egozentrisch motivierte Denkmuster nicht auszukommen scheint und den Menschen als solchen immer und unmittelbar betrifft: Warum fragt in der Debatte niemand, wie sich in der Frage, ob man nun gentechnisch veränderte Pflanzen anbaut oder nicht, das größte Glück der größten Zahl erreichen lässt? Hängt nicht die Beantwortung dieser Frage auch damit zusammen, wie wir den Anbau von gentechnisch veränderten Pflanzen mit einer Vorstellung von Gerechtigkeit zusammenbringen?

Dieser Beitrag diskutiert die Anwendung von gentechnisch veränderten Pflanzen folgenorientiert, also nicht im Sinne eines prinzipiellen „Ob“, sondern im Sinne eines an Anwendungskonsequenzen orientierten „Wie“": ,Whether [...] we articulate the task in utilitarian, in Kantian, or in other terms, the claims of justice and of beneficence for the two cases are similar" (O’Neill 1996, S. 109). Denn außerhalb der Europäischen Union kommen gentechnisch veränderte Pflanzen bereits in hohem Maße zur Anwendung. Und obwohl Anwendung noch kein Argument für Erlaubtheit darstellt, soll hier einmal vom Faktum der Anwendbarkeit ausgegangen werden. Viele der bekannten gentechnisch veränderten Pflanzen dienen direkt oder indirekt der menschlichen Ernährung, und sofern die Zulassung (in der Regel außerhalb der EU) erfolgt ist, werden sie auf Freilandflächen angebaut. Hier ergeben sich Wechselwirkungen zwischen den gentechnisch veränderten Pflanzen und anderen Organismen, und diese Wechselwirkungen betreffen auch den Menschen. Dabei sind nicht nur medizinische Zusammenhänge angesprochen, sondern auch juristische, ökologische, ökonomische und viele mehr.

Unter welchen Bedingungen erscheint der Gebrauch gentechnisch veränderter Pflanzen moralisch gerechtfertigt? Lässt sich durch den Anbau von gentechnisch veränderten Pflanzen 
ein höchster Nutzen für die größte Zahl erreichen? Was ist zu tun, damit die grüne Biotechnologie im besten Sinne jedermann nützt? Erste Hinweise und Antwortversuche hierzu finden sich etwa in Klaus Hahlbrocks anthroporelationalen Kriterien (Hahlbrock 2007, S. 308; vgl. ähnlich Strünck 2006, S. 189):

Erstens: Bei der Anwendung grüner Biotechnologie muss der Erhaltung einer lebensfähigen Biosphäre oberste Priorität zukommen. Damit ist zum einen angesprochen, dass grüne Biotechnologie nicht zur Umweltverschmutzung (etwa durch $\mathrm{CO}_{2}$-Emission) beiträgt, sondern sie im Gegenteil zu verhindern hilft. Zum anderen muss eine Pflanze danach beurteilt werden, inwieweit durch ihren Anbau Pestizide und Herbizide zum Einsatz kommen, die wiederum die Biosphäre ungünstig beeinflussen. Sollten sich bei herbizidtoleranten und insektenresistenten Pflanzen langfristig keine Unempfindlichkeiten einstellen, sind sie bezüglich des Arguments der Biosphäre positiv zu werten. Prinzipiell positiv zu werten ist jede Form der ökologischen, d. h. nichtindustriellen Landwirtschaft, da hier Schädlingsbekämpfungsmittel nur begrenzt eingesetzt werden, was sich jedoch negativ hinsichtlich der Ernteerträge auswirkt.

Zweitens: Durch den Einsatz grüner Biotechnologie muss die menschliche Ernährung in quantitativer und qualitativer Hinsicht sichergestellt sein. Neben Maßnahmen zur Ertragssicherung sind dabei besonders Maßnahmen zur Ertragssteigerung angesprochen, die bislang jedenfalls für gentechnisch veränderte Pflanzen noch nicht beobachtet werden konnten (Sauter 2008, S. 9). Das Problem erfährt zusätzliche Brisanz durch die steigende Nachfrage nach Lebensmitteln (bedingt durch das weltweite Bevölkerungswachstum) bei gleichzeitiger Verringerung der zur Verfügung stehenden landwirtschaftlichen Nutzflächen (Engelmann et al. 1996, S. 8f., 39f.). Hier scheinen gentechnisch veränderte Pflanzen ebenfalls ein Ausweg zu sein, wenn selbst unter extremen klimatischen Voraussetzungen hohe Ernteerträge erwartbar wären. Schließlich muss auch thematisiert werden, zu welchem Zweck eine Pflanze gezüchtet und angebaut wird: Handelt es sich um Pflanzen zur Nahrungsmitteloder zur Energiegewinnung? Hier wäre den Nahrungsmittelpflanzen der Vorrang einzuräumen.
Drittens: Grüne Biotechnologie sollte einen Beitrag zu Vorsorge und Erhaltung der menschlichen Gesundheit leisten. Es muss also wenigstens ein Nachweis erbracht werden, dass Anbau und Verzehr von gentechnisch veränderten Pflanzen keine negativen gesundheitlichen Folgen haben, und dieser Nachweis muss eine langfristige Perspektive abdecken können (van den Daele 1999, S. 266f.). Ergebnis der Langzeittests kann freilich auch die Befürwortung von gentechnisch veränderten Pflanzen sein, insofern sie z. B. Mangel- oder Fehlernährung effektiv und nachhaltig beseitigen können. Weil sich diese Ergebnisse in der Regel mittels konventioneller Züchtungsverfahren nicht erreichen ließen, wäre auch hier im besten Falle die Gentechnik zu befürworten.

Viertens: Grüne Biotechnologie muss für die Beachtung des Artenschutzes in Bezug auf Pflanzen und Tiere sensibilisiert sein. Bei ,konventionellen' Pflanzen stellt sich die Frage, wie die Auswirkungen durch Dünger und Schädlingsbekämpfungsmittel zu beurteilen sind, bei gentechnisch veränderten Pflanzen muss die Frage der Auswilderung und der Auskreuzung und die Frage der Toleranz und Resistenz gegenüber nicht gentechnisch veränderten Organismen reflektiert werden; auch muss dafür Sorge getragen werden, dass sich nicht eine erfolgreiche Sorte monokulturartig durchsetzen kann, sondern dass Biodiversität in gewissem Maße erhalten bleibt.

Fünftens: Beim Vertrieb von mithilfe grüner Biotechnologie hergestellten Produkten ist dafür zu sorgen, dass die Menschenrechte im Allgemeinen geachtet werden. Übergeordnetes Ziel ist dabei der Zugang zu Bildung, landwirtschaftlicher Nutzfläche, Wasser und Saatgut. Auf positive ökonomische Effekte und indirekte Einflüsse auf das regionale Wohlstandsniveau durch gentechnisch veränderte Pflanzen (z. B. Bt-Baumwolle in Indien) weisen Qaim/Subramanian (2010) hin, und es bedarf der Diskussion, inwiefern diese Effekte langfristig anhalten. Zugleich setzt der Anbau von gentechnisch veränderten Pflanzen ein hohes Wissen um die Technik voraus, es müssen bei dem gewählten Saatgut die passenden Komponenten zur richtigen Zeit eingesetzt werden, und dieses Wissen kann bei Kleinbauern in Entwicklungsländern nicht unbedingt vorausgesetzt werden. Hinzu kommt, dass einige gentechnisch veränderten 
Pflanzen nicht für den Einsatz auf kleinen Parzellen angelegt sind, sondern eine großflächige Bewirtschaftung mit einem hohen technischen Einsatz erforderlich machen (HT-Mais). Dabei sind v. a. wirtschaftspolitische Sujets zu problematisieren, besonders die Abhängigkeit der Kleinbauern von Komplettlösungen der Saatgutkonzerne, die den optimalen Mix aus Saatgut, Dünger und Schädlingsbekämpfung exklusiv anbieten und Kleinbauern gelegentlich in eine umfassende Abhängigkeit bringen (was nicht selten den Vorwurf neoimperialistischer Ambitionen einbringt). Diese Tendenz wird durch internationale Abkommen wie TRIPS (Trade-Related Aspects of Intellectual Property Rights) verstärkt, wonach Pflanzenbestandteile durch Patente geschützt werden können, was im Extremfall dazu führen kann, dass Kleinbauern ein traditionelles, indigenes Getreide nicht mehr anbauen dürfen, sondern lizenzpflichtig bestellen müssen. Insbesondere dieser Punkt bedarf einer Diskussion, denn ohne TRIPS verändern zu wollen und zu können, stellt sich doch die Frage, ob man die Rahmenbedingungen nicht so anpassen kann, dass wenigstens die problematischen Aspekte von TRIPS aufgefangen werden.

\section{Thomas Pogge und Health Impact Fund (HIF)}

In einem anderen Kontext, jedoch ebenfalls mit Bezug auf TRIPS, schlagen der Ökonom Aidan Hollis und der Philosoph Thomas Pogge mit dem Health Impact Fund (HIF) ein Modell zum globalen Umgang mit patentgeschützten Medikamenten vor. Der HIF bezeichnet ein System, das Anreize zur Entwicklung und Vermarktung von Medikamenten setzt, die das Interesse an Profitmaximierung seitens der pharmazeutischen Industrie mit den Vorsorge- und Behandlungsbedürfnissen von weniger konsumkräftigen Teilen der Weltbevölkerung verbinden könnten. In Pogges eigenen Worten handelt es sich um einen jährlichen „Forschungswettbewerb, der sich auf alle Länder und alle Krankheiten erstreckt und Forschungsergebnisse gemäß ihrer Gesundheitsauswirkungen belohnt" (Pogge 2011c, S. 276). Das Konzept geht jedoch über einen bloßen Wettbewerb hinaus, denn Pogge verspricht sich von der Initiative (finanzielle) Anreize für pharmazeutische Innovationen, und diese Innovationen würden gemäß ihrer globalen Gesundheitswirkung beurteilt und zum niedrigsten möglichen Verkaufspreis gehandelt (Banerjee et al. 2010, S. 166).

Ausgangspunkt dieser Überlegungen ist die Beobachtung, dass Medikamente in der Regel zu einem hohen Preis verkauft werden. Zwar rechtfertigen die Hersteller ihre Preisgestaltung durch hohe Forschungs- und Entwicklungskosten, nach Ansicht Pogges bleiben jedoch die langfristigen Produktionskosten, die im Normalfall deutlich geringer sind, in der Kalkulation unberücksichtigt. Patente auf Arzneimittel sollen das innovative Produkt schützen und garantieren, dass die Entwicklungskosten durch den Verkauf ausgeglichen werden. Die Folge seien, so Pogge, hohe Gewinne und eine willkürliche Preisgestaltung, und dies sei insofern problematisch, als damit besonders Menschen in Entwicklungsländern vom Zugang zu wirksamer Medizin ausgeschlossen sind: „, billions of human beings are too poor to afford medicines at monopoly prices and thus cannot share the benefit of a patent regime" (Pogge 2011b, S. 245).

Pogges Situationsanalyse lässt sich wie folgt zusammenfassen (vgl. Pogge 2011b, S. 246f.): Patentierte Medizin wird in der Regel unter dem Maßstab der Profitmaximierung verkauft, wodurch die Bewohner der Entwicklungsländer ausgeschlossen, d. h. als Zielgruppe uninteressant werden. Also existieren nur wenige Anreize zur Herstellung kurativ und präventiv wirksamer Medikamente, denn Produkte etwa gegen Haarausfall und Akne sind lukrativer. Zudem dürfte es einen Markt für gefälschte Präparate geben, deren Wirksamkeit nicht gewährleistet ist. Es entstehen unnötige Kosten durch nationale Zulassungsverfahren und durch Marketing, die in die Preisgestaltung der Medikamente einfließen. Schließlich existiert das Problem der letzten Meile, d. h., man weiß aktuell nicht, ob ein Medikament gemäß der Herstellerangaben eingenommen wird. Pogge schlussfolgert deshalb: „the existing international practices and global institutional order must count as unjust and their continued imposition as a harm done to the world's poor" (Pogge 2011b, S. 244).

Die Initiative in Form des HIF reagiert auf diese Analyse (Pogge 2011b, S. 247f.; Pogge 2011c; Banerjee et al. 2010, S. 166f.): Der HIF ist gedacht als eine überstaatliche Einrichtung, die global agiert und durch öffentliche Haushalte fi- 
nanziert wird. Der Hersteller eines Medikaments kann nun sein Produkt (optional) beim HIF registrieren und geht damit die Verpflichtung ein, das Produkt zu dem niedrigsten möglichen Preis (einkalkuliert die Kosten für Produktion und Vertrieb) zu verkaufen. Im Gegenzug erhält er für einen fixen Zeitraum (zehn Jahre) eine Vergütung aus dem Fond, deren Höhe sich an den weltweit in qualitätskorrigierten Lebensjahren (QALY) messbaren Gesundheitswirkungen orientiert. ${ }^{1}$ Nach Ende des Lizenzierungszeitraums müssten die Lizenzen für Herstellung und Handel mit Generika bereitgestellt werden. Die Initiative hat nach Pogge den Vorteil, dass sie pharmazeutische Innovationen fördert, wenig anfällig für Beeinflussung (weil nicht krankheitsspezifisch) ist, Anreize für die optimale Einnahme eines Medikaments setzt, den gesundheitlichen Impact stärker als bisher berücksichtigt und Vergleiche diverser Medikamente zulässt. Weil der HIF eine doppelte Profitabilität fördert (ökonomisch und gesundheitlich), liegt das Interesse des Arzneimittelherstellers v. a. darin, dass viele Menschen von seinen Produkten profitieren, denn der HIF setzt Anreize für die tatsächliche Wirksamkeit von Medikamenten. Es kommen verstärkt die Interessen der Entwicklungsländer in den Blick, da dort viele Menschen an Krankheiten leiden, die zu erforschen derzeit nicht lukrativ erscheint. Werbung für Medikamente wird überflüssig, für Fälschungen gibt es keinen Anreiz, da Medikamente zu günstigen Preisen nach Wirksamkeit verordnet werden. Schließlich muss es im Interesse des Herstellers liegen, seine Medikamente auch sinnvoll eingenommen zu sehen, weil die Zuteilung aus dem Fond davon abhängt. Selbst die Steuerzahler aus den Industrienationen (die für die Anschubfinanzierung sorgen müssten) profitierten letztlich von langfristig niedrigeren Arzneimittelpreisen.

Unabhängig davon, inwieweit das Konzept praktikabel erscheint und welche Probleme sich in der Durchführung ergeben (eine Systematisierung der Kritik an Pogges Modell findet sich z. B. bei Liddell 2010), darf an dieser Stelle die (ethisch relevante) Frage thematisiert werden, ob der HIF nicht auch ein Modell für die Anwendung der grünen Biotechnologie sein kann und ob ein analog zum HIF entworfenes Konzept die strukturelle Ungerechtigkeit in Bezug auf TRIPS und die Anwendung von gentechnisch veränderten Pflanzen überwinden hilft.

\section{Gedankenspiel zur doppelten Profitabilität gentechnisch veränderter Pflanzen}

Ist der HIF anwendbar auf die grüne Biotechnologie? Thomas Pogge selbst regt in anderen Kontexten eine Global Resources Dividend und einen Ecological Impact Fund an, also hält er sein Konzept auch an vergleichbare Sachverhalte anpassbar (Pogge 2010, S. 539-542; Pogge 2011a, S. 336). Kann es eine überstaatliche Regulierung der grünen Biotechnologie geben, wie müsste etwa ein Food Impact Fund oder ein Welfare Impact Fund konkret aussehen? Hahlbrocks anthroporelationale Kriterien (s. o.) könnten hier als Grundlage dienen und mit Blick auf Thomas Pogges HIF eingepasst werden. Die Frage ist, wem die grüne Biotechnologie nützen soll und wie man mit ihr das größte Glück der größten Zahl erreicht, ohne im besten Fall jemanden schlechter zu stellen.

Es ist klar, dass grüne Biotechnologie - und hier im Besonderen die gentechnisch veränderten Pflanzen - an die Erhaltung einer lebensfähigen Biosphäre geknüpft sein muss, dass darüber hinaus die menschliche Ernährung in quantitativer wie in qualitativer Hinsicht gesichert sein muss, dass die menschliche Gesundheit nicht beeinträchtigt werden darf, dass weitere Pflanzen und Tiere nicht zu Schaden kommen dürfen und dass Menschenrechte nicht missachtet werden dürfen. Damit liegen die Analogien auf der Hand: Besonders in den Entwicklungsländern fehlt es $u$. a. an Nahrungsmitteln; die mit TRIPS verbundene Patentierung von pflanzlichem Genmaterial und die in der Folge als Produkt verkaufte gentechnisch veränderte Pflanze schaffen Abhängigkeiten von monopolähnlichen Saatgutkonzernen. Schließlich sind die Kosten für Entwicklung, Vermarktung und Zulassung von gentechnisch veränderten Pflanzen immens hoch, was sich auf den Preis des Produkts auswirkt, solang es durch Patente geschützt ist. Es bietet sich daher an, abschließend ein Modell für den Anbau und die Entwicklung von gentechnisch veränderten Pflanzen zu entwerfen.

Ein möglicher Welfare Impact Fund (WIF) müsste wie auch der HIF global agieren, denn nur in einer globalen Perspektive erweist er sich als 
sinnvoll. Gentechnische oder biotechnologische Innovationen könnten dann ebenso optional registriert werden, was zur Folge hätte, dass für einen festgelegten Zeitraum eine Vergütung aus dem Fond erfolgen müsste. In diesem Zeitraum müsste das Produkt weltweit zum niedrigsten möglichen Herstellungspreis verkauft werden, und die Vergütung aus dem Fonds würde sich nach den Auswirkungen auf das Wohlstandsniveau richten. Am Ende des ,Patentschutzes ' müssten dann die Lizenzen wieder freigegeben werden.

Was ist der Vorteil des Konzepts gegenüber bisherigen Lösungen? Bisher sind Langzeittests v. a. das Mittel der Wahl, ohne den Test ist die Zulassung einer gentechnisch veränderten Pflanze nahezu unmöglich. Der wirtschaftliche Handel mit gentechnisch verändertem Saatgut ist basal durch TRIPS geregelt, unterliegt ansonsten jedoch den Bestimmungen von Angebot und Nachfrage sowie den Produktionsbedingungen einschließlich der wirtschaftlichen Profitabilität. Hier könnte der WIF entscheidende Anreize bezüglich einer doppelten Profitabilität setzen, d. h., es kämen wirtschaftliche und wohlstandsorientierte Gründe ins Spiel. Bei der Ausschüttung aus dem Fond müssten dann Hahlbrocks anthroporelationale Kriterien einbezogen werden: Wie wirkt sich eine gentechnisch veränderte Pflanze betreffend der Biosphäre aus? Welcher Beitrag ist für die qualitative wie quantitative Sicherung der menschlichen Ernährung erreicht worden? Ist die menschliche Gesundheit durch das Produkt gefährdet oder wird sie unterstützt? Ist der Artenschutz im Hinblick auf Tiere und andere Pflanzen gewährleistet? In welchem Maße bleibt Biodiversität erhalten? Sind die Menschenrechte geachtet und berücksichtigt, zum Beispiel mit Blick auf Zugang zu Saatgut und zu Bildung (,Wie baue ich das Produkt an?")? Wird mit dem von mir hergestellten Saatgut auch wirklich ein optimaler Erfolg erzielt? Und die Befriedigung welcher Bedürfnisse verspricht schließlich die effektivste Wirkung auf das weltweite Wohlstandsniveau?

Zugegeben, der hier skizzierte WIF verspricht noch deutlich komplexer zu werden als der von Pogge und Hallis vorgeschlagene HIF. Unmittelbare Auswirkungen auf das Wohlstandsniveau wären schwerlich durch QALYs zu erfassen, Kriterien zur Vergütung aus dem WIF müssten noch ausgehandelt und definiert werden. Zudem würde immer der Verdacht im Raum stehen, der WIF hielte eben nur Regulierung und Bürokratie bereit, könne aber keinen substanziellen Beitrag etwa zur Besserung der Welternährungssituation leisten. Immerhin erlaubt jedoch der WIF als ein Gedankenspiel, Sachverhalte globaler Verteilungsungerechtigkeiten zu benennen und über ihre Beseitigung nachzudenken, ohne den Status quo mehr als nötig infrage zu stellen. Der WIF wäre ein gigantisches Experiment, und er könnte, wenn er funktioniert, zur Lösung einer der relevantesten Fragen des 21. Jahrhunderts beitragen, nämlich zur Frage gleichberechtigter und gerechter Teilhabe am Wohlstand, besonders hinsichtlich der Welternährung.

\section{Anmerkung}

1) Faktoren der Beurteilung sind klinische und anwendungsbezogene Tests und statistische Erhebungen über die Korrelation von Einnahme des Produkts und Entwicklung der Krankheit.

\section{Literatur}

Banerjee, A.; Hollis, A.; Pogge, T., 2010: The Health Impact Fund: Incentives for Improving Access to Medicines, In: Lancet 375/9709 (2010), S. 166-169

Engelmann, R.; LeRoy, P.; Vetter, H., 1996: Mensch, Land! Report über Weltbevölkerungsentwicklung und nachhaltige Nahrungsproduktion. Hannover

Hahlbrock, K., 2007: Kann unsere Erde die Menschen noch ernähren? Bevölkerungsexplosion, Umwelt, Gentechnik. Frankfurt a. M.

Korthals, M., 2003: Grüne Gentechnik. In: Düwell, M.; Steigleder, K. (Hg.): Bioethik. Eine Einführung. Frankfurt a. M., S. 354-362

Kummer, C., 2013: Pflanzenwürde, In: Stimmen der Zeit 138/1 (2013), S. 21-30

Liddell, K., 2010: The Health Impact Fund: A Critique. In: Pogge, T.; Rimmer, M.; Rubenstein, K. (Hg.): Incentives for Global Public Health. Patent Law and Access to Essential Medicines. Cambridge, UK, S. $155-180$

O'Neill, O., 1996: Ending World Hunger. In: Aiken, W.; LaFollette, H. (Hg.): World Hunger and Morality. Upper Saddle River, N.J, S. 85-112

Pogge, T., 2010: Keynote Address: Poverty, Climate Change, and Overpopulation, In: Georgia Journal of 
International \& Comparative Law 38/3 (2010), S. 525-542

Pogge, T., 2011a: Allowing the Poor to Share the Earth, In: Journal of Moral Philosophy 8 (2011), S. 335-352

Pogge, T., 2011b: The Health Impact Fund: How to Make New Medicines Accessible to All. In: Benatar, S.R.; Brock, G. (Hg.): Global Health and Global Health Ethics. Cambridge, S. 241-250

Pogge, T., 2011c: Weltarmut und Menschenrechte. Kosmopolitische Verantwortungen und Reformen. Berlin

Qaim, M.; Subramanian, A., 2010: Benefits of Transgenic Plants: A Socioeconomic Perspective. In: Kempken, F.; Jung, C. (Hg.): Genetic Modification of Plants. Agriculture, Horticulture and Forestry. Berlin, S. 615-629

Sauter, A., 2008: Transgenes Saatgut in Entwicklungsländern. Erfahrungen, Herausforderungen, Perspektiven. Berlin

Strünck, C., 2006: Die Macht des Risikos. Interessenvermittlung in der amerikanischen und europäischen Verbraucherpolitik. Baden-Baden

van den Daele, W., 1999: Von rechtlicher Risikovorsorge zu politischer Planung. Begründungen für Innovationskontrollen in einer partizipativen Technikfolgenabschätzung zu gentechnisch erzeugten herbizidresistenten Pflanzen. In: Bora, A. (Hg.): Rechtliches Risikomanagement. Form, Funktion und Leistungsfähigkeit des Rechts in der Risikogesellschaft. Berlin, S. 259-289

\section{Kontakt}

Dr. Christian Berkenkopf

Lehrstuhl für Dogmatik und Dogmengeschichte

Kath.-Theol. Fakultät

Ruhr-Universität Bochum

GA 7/33, Universitätsstr. 150, 44801 Bochum

Tel.: +49 234 32-22454

E-Mail: christian.berkenkopf@rub.de

$《 》$

\section{Technikethische Werte im Konflikt - Das Beispiel des Körperscanners}

\author{
von Thilo Hagendorff, Internationales \\ Zentrum für Ethik in den Wissenschaften
}

Die Entwicklung technischer Artefakte ist immer von Werten beeinflusst, welche in die Technik eingeschrieben werden, jedoch in der späteren Anwendung der Technik schwierig erkennbar und kaum mehr verhandelbar sind. Exemplifiziert wird dies im folgenden Aufsatz an Körperscannern, einer relativ neuen Sicherheitstechnologie, deren Einsatz nicht-intendierte Nebenfolgen mit sich bringt, welche wesentliche technikethische Fragen aufwerfen. Körperscanner konstruieren durch häufige Fehldetektionen „,auffällige“ oder „,gefährliche" Personen. Fehldetektionen entstehen, wenn die Körper der zu scannenden Personen ,inkompatibel“ sind mit dem seitens der Technik vorgegebenen Normalkörperschema. Werte, welche in diesem Fall die Physiognomie und die Form des menschlichen Körpers betreffen, härten in der Technik aus und machen sich über sie geltend. Technik autorisiert somit die Entmächtigung der mit ihr konfrontierten Personen, was im Endeffekt in der handfesten Verletzung von Persönlichkeitsrechten enden kann.

\section{Technikethische Abwägungsprozesse}

Eine Definition von Technik lautet, Technik bestimme sich durch die Gesamtheit derjenigen Verfahren und Einrichtungen, welche Handlungszusammenhänge derart anreichern, dass Tätigkeiten in ihrer Wirksamkeit gesteigert werden können (Rammert 1999, S. 3f.). Diese Wirksamkeitssteigerungen, mit denen Technologien im Allgemeinen beworben werden, sind jedoch abhängig davon, welchen Standpunkt man ihnen gegenüber einnimmt. Was sich auf der einen Seite als technikbedingte Wirksamkeitssteigerung, als Vereinfachung oder Ermöglichung neuer Fertigkeiten niederschlägt, kann auf der anderen Seite das genaue Gegenteil bedeuten. Während Technologien das Erreichen von Zwecksetzungen vereinfachen können, können sie es ebenfalls 